\section{Space-based gravity tests}

\author{
Clifford M. Will
}

WHAT does the future hold, if anything, for experimental studies of gravitation? This was the central question at a recent conference* held to honour the memory of William M. Fairbank, one of the most enthusiastic and forceful advocates for experimental gravitation, who died last year'. Noted for several discoveries in low-temperature physics, Fairbank turned his attention during the 1960s to the application of cryogenic techniques to precision gravity experiments, and his masses of the Earth and Moon) were verified to accuracies ranging from 1 to 0.01 per cent. And observations of the decaying orbit of the remarkable binary pulsar confirmed the theory's predictions for gravitational-wave damping ${ }^{2,3}$.

For all the painstaking and timeconsuming work that went into the experimental tests of the golden era, they were somehow easy, for they were done at a time when advances in technology (atomic clocks, radio and radar astronomy, space

\begin{tabular}{lll}
\hline & SPACE-BASED TESTS FOR GRAVITY & \\
\hline Experiment & Quantity measured & Accuracy proposed \\
$\begin{array}{l}\text { Satellite test of } \\
\text { equivalence principle }\end{array}$ & $\begin{array}{l}\text { Differential acceleration } \\
\text { of different materials }\end{array}$ & $10^{-17}$ \\
$\begin{array}{l}\text { Optical interferometer } \\
\text { in space }\end{array}$ & Light deflection & $10^{-6}$ \\
$\begin{array}{l}\text { Laser-ranged Earth } \\
\text { satellite }\end{array}$ & Frame-dragging of \\
$\begin{array}{l}\text { Atomic clock } \\
\text { on Solar Probe }\end{array}$ & orbit plane \\
$\begin{array}{l}\text { Microwave interferometer } \\
\text { in space }\end{array}$ & Gravitational redshift & $10^{-3}$ arcsec yr $^{-1}$ \\
$\begin{array}{l}\text { Laser interferometer } \\
\text { in space }\end{array}$ & $\begin{array}{l}\text { Gravity-wave strain } \\
\text { at } 10^{-2} \mathrm{~Hz}\end{array}$ \\
$\begin{array}{l}\text { Gravity gradiometer } \\
\text { in space }\end{array}$ & $\begin{array}{l}\text { Gravity-wave strain } \\
\text { at } 10^{-4} \mathrm{~Hz}\end{array}$ & $10^{-18}$ \\
\hline
\end{tabular}

Stanford laboratory soon became a centre for such work. This included the construction of bar detectors for gravitational waves, attempts to measure the free fall of positrons, and an experiment to measure the einsteinian "dragging of inertial frames' by the rotating Earth using an orbiting gyroscope.

Coincidentally, the conference also came within two months of the 75 th anniversary of Einstein's final formulation of the general theory of relativity. Based on the principle of equivalence, or the equality of free-fall acceleration of different bodies, general relativity accounts for gravity as a consequence of curved spacetime. Although the theory was pretty much in hibernation for its first 45 years, the past 30 have witnessed a renaissance of the subject, not least in the area of experimental gravitation. In fact $1960-80$ was something of a golden era for testing relativity, during which most of the theory's predictions for effects in the Solar System (light deflection, gravitational redshift, retardation of light, the advance of Mercury's perihelion and the equivalence principle for the inertial and gravitational

* Relativistic Gravitational Experiments in Space. Rome, 10-14 September 1990 exploration) met the demands for checking the validity of general relativity, which arose from astronomical discoveries. The necessary experiments could be done with good precision, and so they were.

In contrast, the experiments envisaged for the future require almost heroic advances in technology and precision techniques to reach the required sensitivities. Near Zero, a Festschrift for Fairbank ${ }^{+}$ published in 1988 , neatly characterizes the requirements for many of these experiments: near-zero temperature $\left(10^{-3} \mathrm{~K}\right.$ noise temperature in gravity-wave antennae); near-zero magnetic field $\left(10^{-7}\right.$ gauss in the gyroscope experiment); and nearzero acceleration $\left(10^{-11} \mathrm{~g}\right.$ for a satellite test of the equivalence principle). Why? Because the effects to be measured are also near zero.

Nothing illustrates this better than the gyroscope experiment, denoted Gravity Probe B (GP-B) by NASA, which was described in detail at the meeting (C.W.F. Everitt, Stanford University). The plan is to place a set of four superconductingniobium-coated, quartz gyroscopes into polar Earth orbit to measure the predicted 42 milliarcsecond per year precession of the spin axes to better than 0.5 per cent.
The frame-dragging effect responsible for this precession is a rotation of the axes of local inertial frames relative to distant stars caused by the rotation of the Earth (see figure). The effect is sometimes called gravito-magnetism because it is analogous to the precession of a magnetic moment outside a rotating charged body. Apart from being an important testable prediction of general relativity, it plays a central role in supermassive-black-hole models for the formation of jets in quasars, and the notion that distant matter can induce rotation of local inertial frames has obvious machian overtones. Thus the direct detection of this effect is a goal worth reaching, despite the difficulties. The required precision implies measuring a precession rate which is another near zero: $10^{-17}$ radians per second.

In the $1960 \mathrm{~s}$ and $1970 \mathrm{~s}$, what was needed to achieve this seemed beyond the pale: the 4-cm-diameter gyroscopes would have to be homogeneous and spherical to a few parts in $10^{7}$; the spacecraft carrying the experiment would have to be free of drag (residual accelerations less than $10^{-12}$ $g$ ); an onboard telescope would be needed, capable of providing a reference direction stable to 0.1 milliarcseconds per year; and so on. But recently the GP-B laboratory successfully conducted an Earth-bound test of the integrated gyroscope-cryogenics-telescope system (M. Tater, Stanford University), showing that many of the requirements have now been met and that others will follow once the device reaches the low-gravity environment of space. The current plan calls for an orbiting mission around 1996.

But GP-B is not the only space-based gravitation experiment being planned. Others for the next decade are at various stages of development: some are just conceptual; for others, the hardware is being developed. Their goals include detection of low-frequency gravitational waves (seismic noise restricts Earth-bound detectors to seeking frequencies above 1 hertz), orders-of-magnitude improvement of current tests of general relativity, and probes of higher-order relativistic gravitational effects. Some of the projects

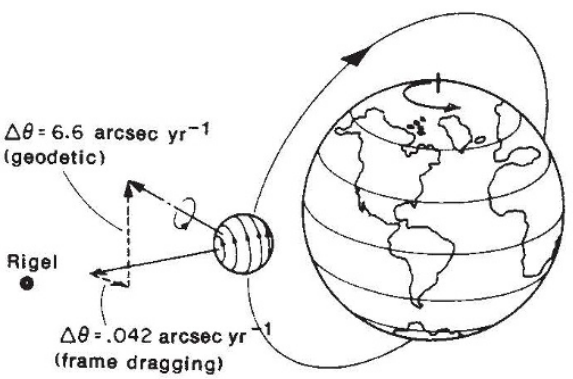

Precession effects due to relativity in a gyroscope experiment orbiting the Earth. Detecting the 42 milliarcsecond change each year due to 'frame dragging' will require remarkable, but achievable precision. (From C.W.F. Everitt in ref. 4, page 589.) 
discussed at the meeting are outlined in the table.

If these experiments are so difficult and take so long, and require access to space (which has lately become such a precious commodity), why bother to do them? The answer, in the case of gravity-wave detectors, is that a new astronomical window will open up, revealing information about violent and dynamic cosmic events. Furthermore, only space-based detectors can 'see' the known sources of gravity waves, such as close binary systems.

As for the basic notion of gravitational tests, general relativity is a fundamental theory of nature, and should be given the best empirical backing we can provide. More importantly, its predictions are fixed; the theory contains nothing that can be adjusted to accommodate any experi- mental deviations. Thus any test of general relativity is potentially a deadly test, for any verified deviations would kill the theory. As his quest for fractionally charged free quarks showed, Fairbank loved to try experiments that might overthrow conventional wisdom; one suspects that even an outside chance of demolishing general relativity would have kept him in the game for many years to come.

Clifford M. Will is in the McDonnell Center for the Space Sciences, Department of Physics, Washington University, St Louis, Missouri 63130, USA.

\section{Cabrera, B. Nature 342, 125 (1989). \\ 2. Will, C.M. Phys. Rep. 113, 345 (1984)}

3. Wili, C.M. Was Einstein Right? (Basic Books, New York, 1986)

4. Fairbank. J.D. et al. (eds) Near Zero: New Frontiers of Physics (W.H. Freeman, New York, 1988).

\section{The race for resistance genes}

\section{Geoffrey North}

Plants have defence mechanisms by which they can resist infection by potentially pathogenic microorganisms. Like the immune systems of higher animals, these mechanisms work by first recognizing foreign molecules produced by the invading pathogen. The recognition repertoire of plants, however, lacks the developmental flexibility of animal immune systems, and is set for the life of a particular plant by the collection of socalled 'resistance' genes it happens to inherit. The cloning of resistance genes is an important goal in plant pathology, and on the evidence of a recent meeting* it should not be long before it is achieved.

Resistance genes commonly exhibit what are known as 'gene-for-gene' interactions with specific genes carried by the pathogen, which are perhaps misleadingly referred to as 'avirulence' genes. Genefor-gene interactions are recognized by a simple pattern of inheritance such that plants carrying a particular resistance gene can recognize, and are thus resistant to, pathogens carrying the complementary avirulence gene, but if either resistance gene or avirulence gene is absent a virulent infection ensues. The resistance gene is assumed to code for some kind of receptor molecule which signals the presence of the product of its complementary avirulence gene, setting off a 'hypersensitive reaction' by which the plant can isolate the pathogen at the site of infection and prevent its spread throughout the plant.

Avirulence genes are thus named because their presence prevents virulent infections on host plants carrying the

* The 5 th International Symposium on the Molecular Genetics of Plant-Microbe Interactions, Interlaken, Switzerland, 9-14 September 1990. appropriate resistance genes. It is presumed that pathogens do not carry avirulence genes to alert host plants to their presence, and work on the pathogen Xanthomonas campestris, which causes bacterial spot disease of tomato and Capsicum, provides evidence that at least some avirulence genes are required for full virulence of the pathogen on susceptible hosts (Kearny, B. \& Staskawicz, B. J. Nature 346, 385-386; 1990).

This property may explain the high degree of conservation of the avirulence gene avrBs 2 among $X$. campestris strains, and why its complementary resistance gene $B s 2$ provides stable field resistance to bacterial spot disease in Capsicum (B. J. Staskawicz, University of California, Berkeley). The hope is that the Bs2 gene will work similarly in other crop plants, to which it could be transferred when it has been cloned.

A number of routes are being taken to clone plant resistance genes in the absence of a defined biochemical product. For example, efforts are being made to exploit the advantages of the small crucifer Arabidopsis thaliana, currently the favourite subject of the plant molecular geneticist (F. Ausubel, Massachusetts General Hospital, Boston). Certain Arabidopsis lines have resistance genes corresponding to avirulence genes carried by particular strains of Pseudomonas syringae, and as a first step Ausubel and colleagues are attempting to isolate mutants that have lost resistance. In particular, they would like to obtain plants with deletion mutations in a resistance gene, which would enable them to apply the technique of 'subtractive hybridization'. Here, genomic DNA with the deletion mutation is hybridized to DNA from the wild-type parental plants in the hope that the nonhybridizing DNA, which can be cloned, would be enriched in sequences representing the deletion. The technique has already been used to clone an Arabidopsis gene for an enzyme of gibberellin biosynthesis; the problem, however, is the lack of a straightforward general way of inducing deletion mutations. Using a screen with the chemical mutagen ethylmethanesulphonate, an Arabidopsis mutant has been obtained that has lost resistance to a particular strain of $X$. campestris (M. J. Daniels, John Innes Institute, Norwich). But cloning the site of mutation is not an easy step.

A subtractive hybridization procedure is also being used to clone tomato resistance genes to the fungal pathogen Cladosprium fulvum (M. Dickinson, John Innes Institute). In this case the subtraction is done not with genomic DNA but with complementary DNA copied from the messenger RNA of plants with and without a particular resistance gene. A number of cDNAs have been identified that seem to be specifically expressed in tomato plants carrying resistance gene $C f 2$, though as yet none has been shown to be the product of the $C f 2$ gene.

Other groups are using an approach well known to human geneticists - that of mapping resistance genes relative to polymorphic markers, followed by 'chromosome walking' to the resistance gene. One requirement is a dense enough map of polymorphic markers throughout the genome, and a restriction fragment length polymorphism (RFLP) map is being built up for lettuce and used to map the position of resistance genes to the downy mildew fungus Bremia lactucae (R. Michelmore, University of California, Davis). A similar approach is being used to map and ultimately clone tomato resistance genes to Pseudomonas syringae pv tomato (S. Tanksley, Cornell University). Both of the groups concerned reported how the construction of a map of polymorphic markers is being facilitated by the use of a new technique based on the polymerase chain reaction (PCR). This simply involves the cloning by PCR of regions of the genome picked out by random primers: several regions are picked out by any one set of primers, thus increasing the chance of finding a polymorphism relative to a search using a single-copy probe.

The practical benefits of cloning resistance genes are likely to be considerable. Moreover the enterprise will probably provide insight into the poorly understood processes of signal reception and transduction in plants, as well as into the evolution of resistance in the arms-race between plants and their pathogens.

Geoffrey North is Deputy Biology Editor of Nature. 